\title{
Viabilité et performance de germination des semences de Acacia auriculiformis A. Cunn. ex Benth. (Fabaceae) issues du sol de la forêt classée de Pahou (Sud Bénin)
}

\author{
G. C. R. Gbaguidi,
}

Centre d'Etudes, de Recherches et de Formation Forestières (CERF), Ministère du Cadre de Vie et du Développement Durable (MCVDD),

République du Bénin

A. K. G. Djotan,

Laboratoire des Sciences Forestières (LSF), Faculté des Sciences Agronomiques (FSA), Université d'Abomey Calavi (UAC), République du Bénin, Laboratory of Forest Botany, Department of Forestry, Graduate School of Agricultural and Life Sciences, University of Tokyo, Japan

\section{G. S. Akouehou, S. R. Lokossou,}

Centre d'Etudes, de Recherches et de Formation Forestières (CERF), Ministère du Cadre de Vie et du Développement Durable (MCVDD),

République du Bénin
J. C. Ganglo,

Laboratoire des Sciences Forestières (LSF), Faculté des Sciences Agronomiques (FSA), Université d'Abomey Calavi (UAC), République du Bénin

\section{Résumé}

La récolte des semences de Acacia auriculiformis devient très difficile lorsque l'arbre prend de l'âge. Cette étude a évalué la viabilité et la performance de germination des semences de cette espèce éventuellement conservées dans le sol. À cet effet, une expérimentation a été faite sur une période de sept semaines pour dénombrer le nombre de plantules en émergence à partir des échantillons composites de sol collectés dans une vieille plantation de Acacia auriculiformis au Sud Bénin. L'échantillonnage est stratifié, aléatoire sur le plan horizontal (provenance), mais systématique sur le plan vertical (profondeur). Les échantillons ont été mis en conditions minimales de germination. La méthode de germination a été utilisée pour évaluer la viabilité et la performance de germination des semences. 
L'indépendance de ces paramètres vis-à-vis des facteurs considérés a été testée avec Khi-2. Enfin, les tendances observées ont été expliquées par les modèles GLM. La profondeur et la provenance se révèlent avoir des influences significatives sur les paramètres mesurés. Le sol de la plantation peut contenir jusqu'à 267 semences viables de Acacia auriculiformis par $\mathrm{m}^{2}$ surtout à l'intérieur de la plantation dans la première couche du sol. Il est possible de faire recours à ces semences du sol pour pallier les difficultés liées à l'approvisionnement en plants au Bénin.

Mots-clés: Peuplement de Acacia auriculiformis, Banque de graines du sol, Production de plantules, Pahou, Sud-Bénin 


\title{
Viability and germination performance of seeds of Acacia auriculiformis A. Cunn. ex Benth. (Fabaceae) from Pahou forest reserve's seed bank (Southern Benin)
}

\section{G. C. R. Gbaguidi,}

Centre d'Etudes, de Recherches et de Formation Forestières (CERF), Ministère du Cadre de Vie et du Développement Durable (MCVDD), République du Bénin
A. K. G. Djotan,

Laboratoire des Sciences Forestières (LSF), Faculté des Sciences Agronomiques (FSA), Université d'Abomey Calavi (UAC), République du

Bénin, Laboratory of Forest Botany, Department of Forestry, Graduate

School of Agricultural and Life Sciences, University of Tokyo, Japan

\section{G. S. Akouehou, \\ S. R. Lokossou,}

Centre d'Etudes, de Recherches et de Formation Forestières (CERF), Ministère du Cadre de Vie et du Développement Durable (MCVDD),

République du Bénin

\section{J. C. Ganglo,}

Laboratoire des Sciences Forestières (LSF), Faculté des Sciences Agronomiques (FSA), Université d'Abomey Calavi (UAC),

République du Bénin

\begin{abstract}
The harvest of the seeds of Acacia auriculiformis becomes very difficult when the tree gets older. This study has assessed the viability, and the germination performance of the species' seeds eventually conserved in the soil. For this purpose, an experiment was carried out over a period of seven weeks to count the number of seedlings emerging from the composite soil samples collected in an old plantation of Acacia auriculiformis in South Benin. Samples were randomly collected over a horizontally stratified scale (provenance), but systematically collected over a vertically stratified scale (depth). The samples have been set to minimum conditions of germination. Germination method was used to assess the viability and the germination performance of seeds. The independence of those parameters with regard to considered factors was tested with Khi-2. Finally, GLM models were used to explain the recorded trends. Depth and provenance seem both to significantly
\end{abstract}


influence assessed parameters. Soil from the plantation can hold up to 267 viable seeds of Acacia auriculiformis per $\mathrm{m}^{2}$ especially inside the plantation in the first layer of the ground. It is possible to rely on those soil's seeds to alleviate the difficulties related to seedling supply in Benin.

Keywords: Acacia auriculiformis stand, Soil seeds Bank, Seedlings production, Pahou, Southern Benin

\section{Introduction}

La production de bois énergie à partir des essences forestières à croissance rapide permet de satisfaire les besoins des populations en bois énergie (Waylen, 2006). Ainsi, les pressions qui pèsent sur les forêts naturelles seront réduites (IPCC, 2007), ces forêts qui ne sont rien d'autre que de maigres ressources en perpétuelle diminution (Ganglo et de Foucault, 2006). Au Bénin, de nombreuses essences forestières telles Gmelina arborea Roxb. Lamiaceae, Khaya senegalensis (Desr.) \& Juss. Meliaceae, Tectona grandis L.f. Lamiaceae, Terminalia superba Engl. Combretaceae, Leucaena leucocephala (Lam.) Fabaceae, Cassia siamea (Lamk.) Fabaceae, etc., sont utilisées. Parmi elles, Acacia auriculiformis est beaucoup plus prisée aussi bien par les privés que par l'Etat dans les plantations destinées à la production de bois énergie à cause de sa vitesse de croissance (Fonton et al., 2002a ; Fonton et al., 2002b) et d'accumulation de biomasse, ainsi que la qualité du charbon que l'on peut obtenir de la transformation de son bois. Acacia auriculiformis a une forte production avec, à six ans, une biomasse sur pied de $125 \mathrm{t} / \mathrm{ha}$ de matière dont 15,6 t/ha d'émondes constituées de petites branches et de pseudo feuilles (Fonton et al., 2002b) fertilisant le sol (Zakra et al., 1996). Des méthodes de prétraitement ont été expérimentées sans aucune germination optimale (Clemens et al., 1977). Cependant, le problème que la mise en plantation de cette espèce rencontre concerne essentiellement la levée de dormance de ses graines et la disponibilité de semences pour la production de plants. De nombreuses techniques et méthodes de levée de dormance ont été décrites dans la littérature par de nombreux auteurs (Myers 1936 ; Aveyard, 1968 ; Brown and Booysen, 1969). Néanmoins, ces méthodes ne sont qu'une partie de la solution et la production de plants de Acacia auriculiformis reste un grand défi.

En effet, Acacia auriculiformis fructifie très abondamment dès sa deuxième année (Gnahoua \& Louppe, 2003) mais, la récolte des semences devient de plus en plus difficile au fur et à mesure que l'âge de la plantation augmente. Les difficultés concernent la taille, la conformation de l'arbre et le matériel de récolte qui devient inapproprié, conduisant à un problème de manque de semence. Le problème d'indisponibilité de semences est d'autant plus critique qu'il arrive des moments où il est impossible aux fournisseurs de 
semences (cas par exemple du Centre d'Etudes de Recherche et de Formation Forestières -CERF- au Bénin en 2016) de satisfaire la demande en semences de certaines institutions forestières chargées du reboisement (cas par exemple de la Direction Générale des Eaux, Forêts et Chasse du Bénin -DGEFC- en 2016). Ceci constitue des freins pour les actions et volontés des gouvernements (Akpona et al., 2016) à freiner les pressions qui pèsent sur les forêts tropicales à cause des extractions illégales de bois sans aucune mesure d'accompagnement de la régénération ou de replantation (Opuni-Frimpong et al., 2008).

La présente étude s'intéresse d'une part à la possibilité de produire des plants de Acacia auriculiformis sans avoir ni à appliquer les multitudes techniques et méthodes de levée de dormance, ni à chercher péniblement et parfois sans succès, des semences de l'espèce. D'autre part, elle envisage de proposer des stratégies de production des plants de cette espèce de sorte à rendre disponible en tout temps, les plants à utiliser pour les reboisements. Pour ce faire, un dispositif d'échantillonnage a été installé dans les plantations de Acacia auriculiformis du périmètre de reboisement de Pahou (Sud Bénin) pour évaluer la viabilité et la performance de germination des semences de cette espèce éventuellement conservées dans le sol. Vu que l'espèce produit suffisamment de biomasse (Fonton et al., 2002b), l'hypothèse qui soutient cette recherche est qu'il est possible que la litière et le sol du sous-bois protègent la semence dans le sol, lui permettant de germer facilement lorsque les conditions sont favorables.

\section{Matériel et méthodes \\ Matériel biologique}

La présente étude a porté sur Acacia auriculiformis A.Cunn. ex Benth., une espèce de la famille des Fabaceae. Elle est originaire du Nord de l'Australie, de Papouasie Nouvelle-Guinée et d'Indonésie (Gnahoua \& Louppe, 2003). C'est une espèce qui a été largement introduite et plantée de par le monde tropical et subtropical : Inde, Asie du Sud-Est, Afrique, Amérique du Sud et même en Floride (ICRAF, 2006). Ainsi qu'ailleurs comme en Cambodge, Cameroun, Chine, République Démocratique du Congo, Inde, Japon, Kenya, Malawi, Malaisie, Nigeria, Philippines, Tanzanie, Thailande, Uganda, Zanzibar, Zimbabwe (ICRAF, 2006). On la trouve entre le niveau de la mer et $1000 \mathrm{~m}$ d'altitude. Elle est résistante à la sécheresse, pouvant se contenter d'une pluviosité de 800 (600) mm par an mais supportant aussi 2500 (3500) mm de pluies d'été (Gnahoua \& Louppe, 2003). Les températures annuelles moyennes de son aire d'origine sont supérieures à $26^{\circ} \mathrm{C}$. Les températures inférieures à $15^{\circ} \mathrm{C}$ ne lui conviennent pas, bien qu'elle puisse supporter une gelée occasionnelle (ICRAF, 2006). Elle s'adapte à une grande variété de sols allant des sols sableux aux sols argileux et aux sols à 
hydromorphie temporaire (Fonton et al., 2002a). Elle supporte une certaine salinité et des pH de 3,0 à 9,5 (ICRAF, 2006).

\section{Milieu d'étude}

L'étude s'est déroulée dans la plantation la plus âgée de Acacia auriculiformis ( 7 ans) étendue sur une superficie de 17,95 ha dans le périmètre de reboisement de la forêt classée de Pahou. La forêt classée de Pahou couvre une superficie de 765 hectares (Akouehou et al., 2009). Elle est située au Sud Bénin dans 1'Arrondissement de Pahou, Commune de Ouidah, dans le département de l'Atlantique, entre les latitudes $6^{\circ} 22^{\prime}$ et $6^{\circ} 27^{\prime}$ Nord et les longitudes $2^{\circ} 8^{\prime}$ et $2^{\circ} 14^{\prime}$ Est. La figure 1 présente le milieu d'étude. Ce domaine appartient à la zone climatique humide (Sinsin et al., 2004; HarvestChoice, 2010) dite zone Guinéo-Congolaise (White, 1986). Dans cette zone, on observe deux saisons pluvieuses (mi-mars à mi-juillet et septembre à mi-novembre) séparées par des périodes sèches, avec une pluviométrie maximale annuelles d'environ $1110 \mathrm{~mm}$ (Adomou, 2005, Neuenschwander et al., 2011). L'humidité relative de l'air varie de $60 \%$ en période d'harmattan (janvier et février) à 95\% au pic de la saison pluvieuse en juin (PAP, 2010)). La température moyenne journalière oscille faiblement autour de $27^{\circ} \mathrm{C}$. Le mois de mars est le plus chaud. On enregistre une température de $28,25^{\circ} \mathrm{C}$, alors que la période juillet - août est celle où les températures sont les plus basses avec une moyenne de $25^{\circ} \mathrm{C}$ (PAP, 2010). La forêt repose sur un sol variant de sableux à sablo-argileux (Volkoff \& Willaime, 1976).

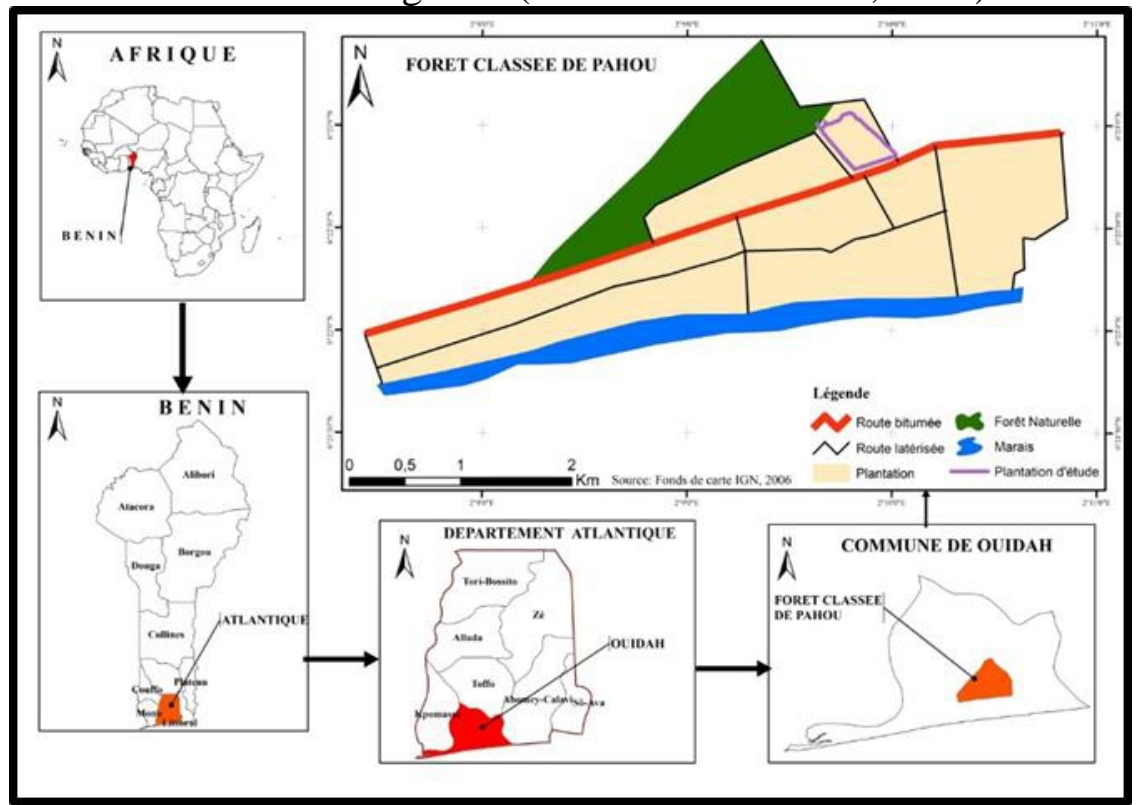

Figure 1 : Localisation de la zone d'étude 


\section{Echantillonnage et collecte de données}

Deux facteurs ont été pris en compte lors de l'échantillonnage. Sur le plan horizontal, c'est-à-dire la provenance (Figure 2a), l'échantillonnage était aléatoire et stratifié avec trois strates (l'intérieur de la plantation, et deux bandes larges chacune de $10 \mathrm{~m}$ autour de la plantation). Sur le plan vertical, c'est-à-dire la profondeur (Figure 2c), l'échantillonnage était systématique et stratifié avec trois strates (couche de profondeur 0-5 cm, 5-10 cm, puis 10-20 $\mathrm{cm}$ ). La méthode de Bauduin (2009) a été utilisée, mais avec l'observation d'une distance minimale de $80 \mathrm{~m}$ entre deux points d'échantillonnage compte tenu de la superficie de la plantation. Chaque échantillon de sol est composite, c'est-à-dire issu du mélange de trois prélèvements unitaires rassemblés (Figure 2b), réalisés sur les sommets d'un triangle équilatéral de $1 \mathrm{~m}$ de côté (Perera, 2005 ; Daïnou et al., 2011). Ce triangle est formé de sorte que le point de prélèvement correspondant, soit son centre (Photo 1). L'avantage des échantillons composites réside dans le fait qu'ils tiennent mieux compte de l'hétérogénéité des banques de semences (Swaine, 2001 ; Perera, 2005). Une tarière (Photo 2) de dimensions $10 \mathrm{~cm} \mathrm{X} 10 \mathrm{~cm}$ de cotés et $5 \mathrm{~cm}$ de profondeur a été utilisée.

Au total, 60 échantillons de sol ont été collectés à partir de 20 points échantillons (20 points x 3 profondeurs). La surface d'échantillonnage à chaque point de prélèvement est de $300 \mathrm{~cm}^{2}(10 \mathrm{~cm} \mathrm{X} 10 \mathrm{~cm} \mathrm{X} \mathrm{3),} \mathrm{donc} \mathrm{une}$ surface totale de $6000 \mathrm{~cm}^{2}$ soit $\left(300 \mathrm{~cm}^{2}\right.$ X 20) a été échantillonnée dans la présente étude. Quant aux volumes de sol prélevé, il était de $1500 \mathrm{~cm}^{3}$, soit $(10 \mathrm{~cm} \mathrm{X} 10 \mathrm{~cm}$ X $5 \mathrm{~cm} \mathrm{X} \mathrm{3)} \mathrm{par} \mathrm{point} \mathrm{pour} \mathrm{les} \mathrm{échantillons} \mathrm{des} \mathrm{deux}$ premières couches, et de $3000 \mathrm{~cm} 3$ soit $(10 \mathrm{~cm} \mathrm{X} 10 \mathrm{~cm} \mathrm{X} 10 \mathrm{~cm} \mathrm{X} \mathrm{3)} \mathrm{pour} \mathrm{la}$ couche la plus profonde. Ainsi un volume total de $120000 \mathrm{~cm}^{3}$, soit $\left(1500 \mathrm{~cm}^{3}\right.$ X 2 X $20+3000 \mathrm{~cm}^{3}$ X 20). La figure 2 cidessous illustre les dispositifs d'échantillonnage. Les données ont été collectées le 21 Avril 2018 et les échantillons de sol ont été mis en conditions minimales sous serre (Photo 3), le 22 Avril 2018. Les échantillons de sol ont été disposés dans des récipients en plastique, plus ou moins plats et larges, de sorte que la profondeur du sol répandu à l'étalage ne dépasse pas $2 \mathrm{~cm}$ (Photo 4). Ces échantillons sont arrosés matin et soir jusqu'à la fin de l'expérimentation. A chaque date, le nombre de plantules qui en émergeait était compté. A chaque échantillon a été associée une étiquette renseignant sur les modalités des deux facteurs considérés, la provenance (Intérieur de la plantation soit $\mathrm{I}$, première bande de 0 à $10 \mathrm{~m}$ de la lisière soit $\mathrm{B} 1$, et la deuxième bande de 10 à $20 \mathrm{~m}$ de la lisière soit $\mathrm{B} 2)$ et la profondeur $(0-5 \mathrm{~cm}$ soit $\mathrm{P} 1,5-10 \mathrm{~cm}$ soit $\mathrm{P} 2$, et $10-20 \mathrm{~cm}$ soit $\mathrm{P} 3$ ). 


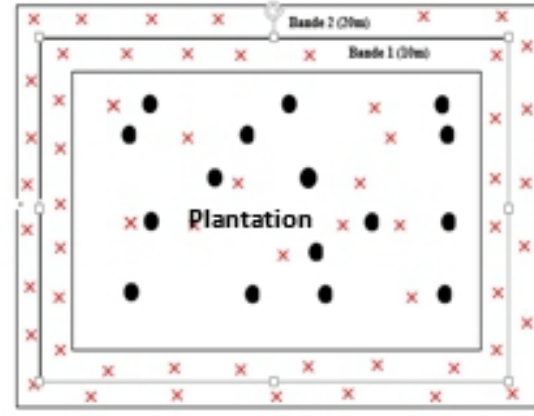

a

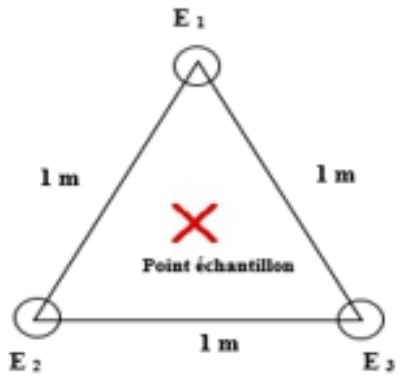

b

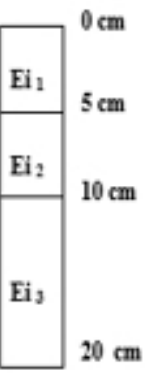

C

Légende :

$X$ : Point échantillon

- : Pied de Acacia

Figure 2 : Illustration des dispositifs d'échantillonnage

\section{Traitement et analyse des données}

La viabilité des semences réfère à leur capacité à germer après être mises dans de conditions minimales de germination, alors que la performance de germination réfère au nombre, c'est-à-dire la quantité de semences qui ont germé dans ces mêmes conditions. Le test d'indépendance Khi-deux de Pearson a été fait pour tester la dépendance de la viabilité des semences du sol vis-à-vis d'une part de la provenance et d'autre part de la profondeur puis le modèle linéaire généralisé (famille négative binomiale) a ensuite été utilisé pour expliquer cette dépendance. Quant à la performance de germination des semences, elle a été estimée à partir de la méthode de germination (Sousa $e t$ al., 2017 ; Douh et al., 2018). Les paramètres utilisés pour décrire l'abondance de semence sont la densité absolue $\left(\mathrm{Da}=\right.$ semences $\left./ \mathrm{m}^{2}\right)$ et la fréquence relative (proportion en \% des échantillons contenant des semences viables), utilisées également par Daïnou et al. (2011) et ; Douh et al. (2018). La performance de germination des semences viables par unité de surface a été expliquée par le modèle linéaire généralisé (famille de Poisson). MS Excel 2013 a été utilisé pour la gestion des données et la représentation des données sous formes de graphiques, mais toutes les analyses statistiques ont été faites dans R (R Core Team, 2016). 


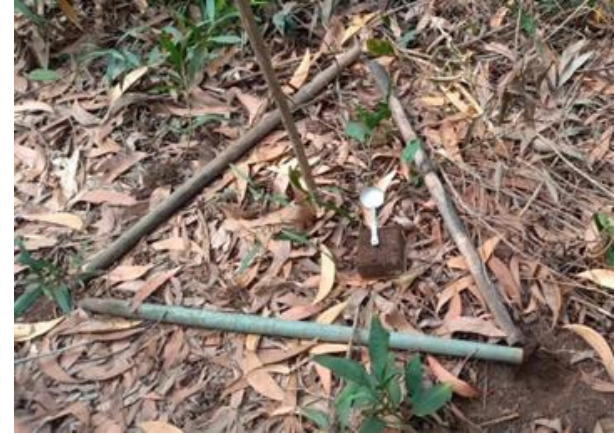

Photo 1: Triangle du point échantillon

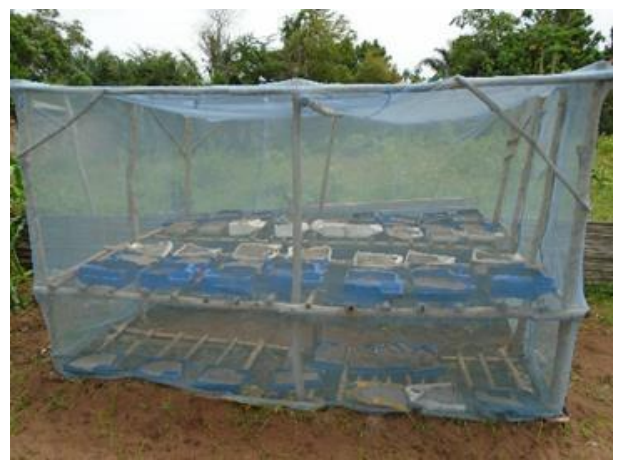

Photo 3: Conditions de germination

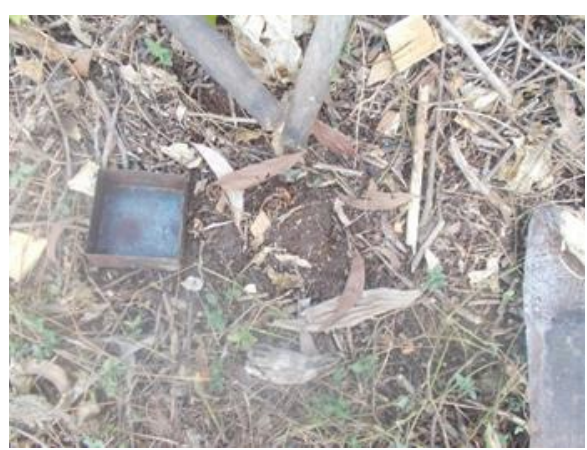

Photo 2: Tarière

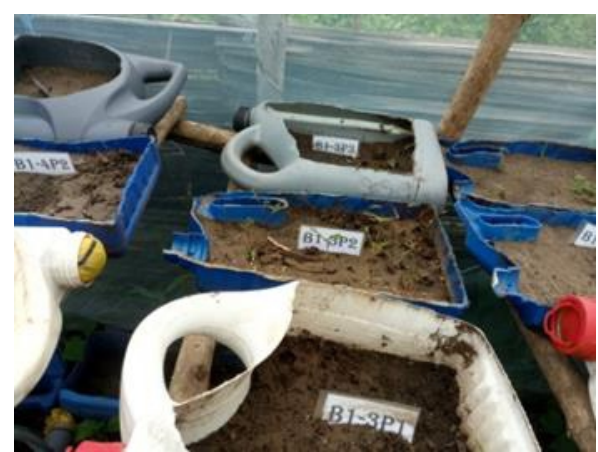

Photo 4: échantillons de sol étiquetés

\section{Resultats et Discussion}

\section{Les semences de Acacia auriculiformis sont viables dans le sol}

Les premières plantules étaient obtenues au bout de deux semaines (14 jours). Le pic de germination était observé quelle que soit la profondeur à la troisième semaine (21 jours), et il n'y avait plus de nouvelles germinations après cinq semaines. Il a été observé au total 167 semences viables, donc 167 plants ont été produits durant l'expérimentation. Tous les échantillons avaient produit de plants, donc possédaient de semences viables. Ainsi la fréquence relative était de $100 \%$.

Ces résultats obtenus montrent que le sol conserve les semences de Acacia auriculiformis, et lorsque ces semences sont mises en conditions minimales de germination, la dormance est levée puis elles germent. Les conditions favorables de germinations remplies par la présente étude étant les minimales, cette dernière témoigne de la probable facilité à produire des plants de Acacia auriculiformis à partir des échantillons de sol. Le sol forestier est donc un conservateur par excellence des semences forestières dont celles de Acacia auriculiformis. Douh et al. (2018) ont déjà rapporté que la banque de semence du sol forestier présente un potentiel de régénération car ces semences ont la capacité de germer. Notons aussi que Schwienbacher et al. (2010) ont déjà trouvé pour des espèces alpines que la longévité des semences 
dans le sol dépend de leur taille et de leur morphologie. Les semences de petite taille et de forme sphérique y en sont dotées d'une grande durée de vie. Les fruits de Acacia auriculiformis sont des gousses plates portant environ 15 graines. Ces graines sont ovales, légères et très petites où environ 30.000 à 72.000 graines pèsent un kilogramme (Gnahoua \& Louppe, 2003). Ces caractéristiques pourraient être valables pour expliquer la capacité du sol à conserver les semences de Acacia auriculiformis. Il a été reconnu que les graines se conservent aisément car possédant une dormance tégumentaire qu'il faut lever à partir de l'eau bouillante, de l'acide sulfurique ou du feu pour avoir une germination rapide et régulière (Gnahoua \& Louppe, 2003). Cependant, notre étude a montré que juste à partir d'un prélèvement de sol dans une plantation de Acacia auriculiformis à une profondeur comprise entre $0 \mathrm{~cm}$ et $5 \mathrm{~cm}$, on peut produire jusqu'à 267 plants par $\mathrm{m}^{2}$ (Tableau 1). Toutefois, il est important de répéter la même expérimentation en variant le milieu d'étude et en testant aussi l'âge de la plantation.

Viabilité et performance de germination des semences d'Acacia auriculiformis dans le sol forestier sous couvert végétal

Les figures $3 \mathrm{a}, \mathrm{b}$ et $\mathrm{c}$ ci-dessous présentent l'évolution du nombre moyen de germinations obtenues par provenance et profondeur au cours de l'expérimentation.

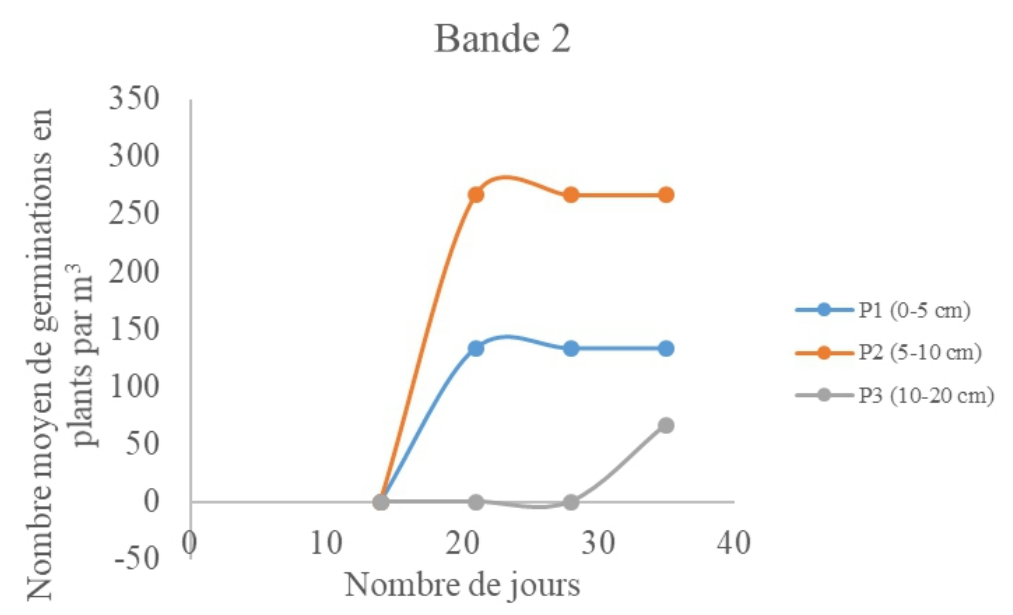

Figure 3a : Évolution du nombre moyen de germinations des semences issues des échantillons de la bande 2 


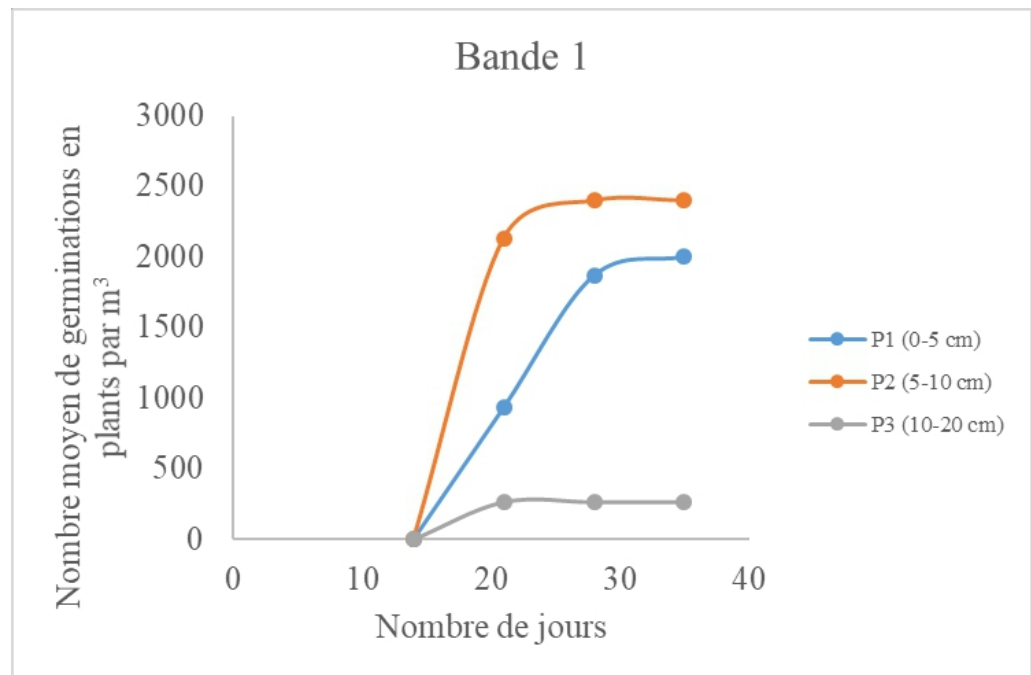

Figure 3b : Évolution du nombre moyen de germinations des semences issues des échantillons de la bande 1

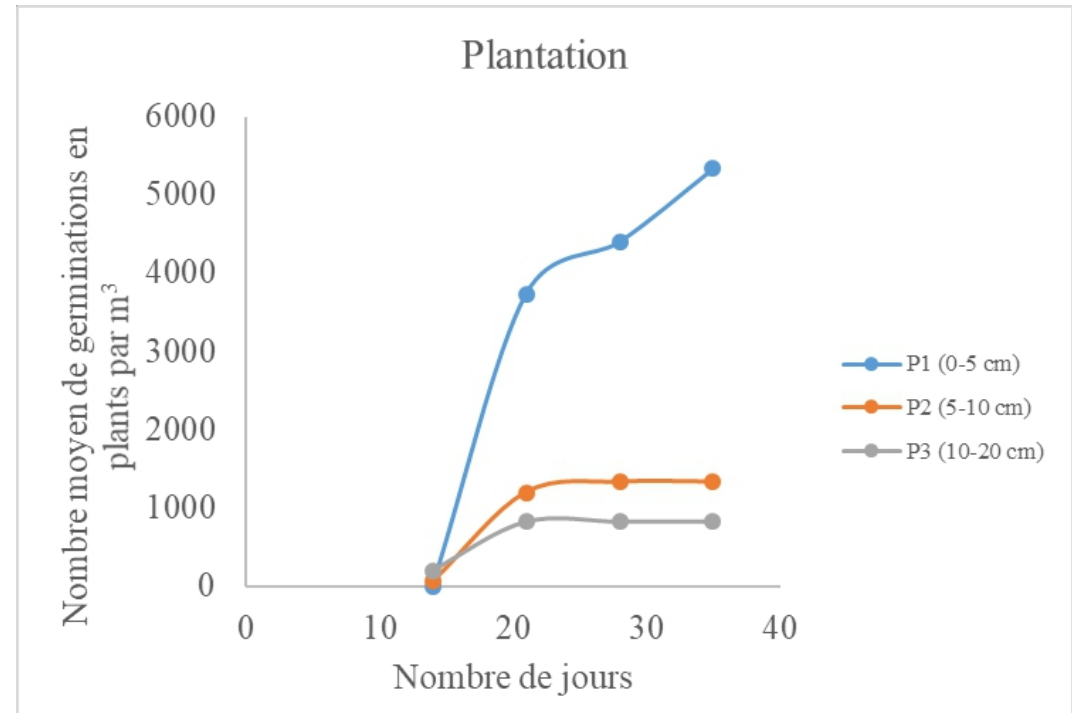

Figure 3c : Évolution du nombre moyen de germinations des semences issues des échantillons de l'intérieur de la plantation

Il ressort de l'analyse des figures $3 \mathrm{a}$, $\mathrm{b}$ et $\mathrm{c}$ que la contribution de la profondeur à la germination des semences de Acacia auriculiformis issues du sol varie d'une provenance à une autre. Le test d'indépendance Khi-deux de Pearson a confirmé que la germination des semences dépend de leur provenance $(P$-value $=0,0001904)$, mais elle est indépendante de la profondeur où se trouve la semence $(P$-value $=0,3999)$. La profondeur $3(10-20 \mathrm{~cm}) \mathrm{s}$ 'est révélée quelle que soit la provenance, contenir très peu de semences viables de Acacia auriculiformis. Dans la plantation, la profondeur $1(0-5 \mathrm{~cm}) \mathrm{s}$ 'est 
révélé contenir la plus grande quantité de semences viables de Acacia auriculiformis. Quant aux Bandes 1 et 2, c'est la profondeur 2 qui s'est révélée contenir la plus grande quantité de semences viables de l'espèce.

Le tableau 1 et la figure 4 ci-dessous présentent les densités moyennes de germination obtenues par provenance et par horizon de sol.

Tab1. Densité de germination par couple de facteurs étudiés (en semences par $\mathrm{m}^{2}$ )

\begin{tabular}{ccccc}
\hline \multirow{2}{*}{ Provenances } & \multicolumn{3}{c}{ Profondeurs } & \multirow{2}{*}{ Total } \\
\cline { 2 - 4 } & P1 & P2 & P3 & \\
\hline B1 & 100 & 120 & 26,67 & 246,67 \\
\hline B2 & 6,67 & 13,33 & 6,67 & 26,67 \\
\hline I & 266,67 & 66,67 & 83,33 & 416,67 \\
\hline Total & 373,34 & 200 & 116,67 & 690,01 \\
\hline
\end{tabular}

I, B1 et B2 représentent respectivement l'intérieur de la plantation, la bande à $10 \mathrm{~m}$ de la lisière et la bande à $20 \mathrm{~m}$ de la lisière.

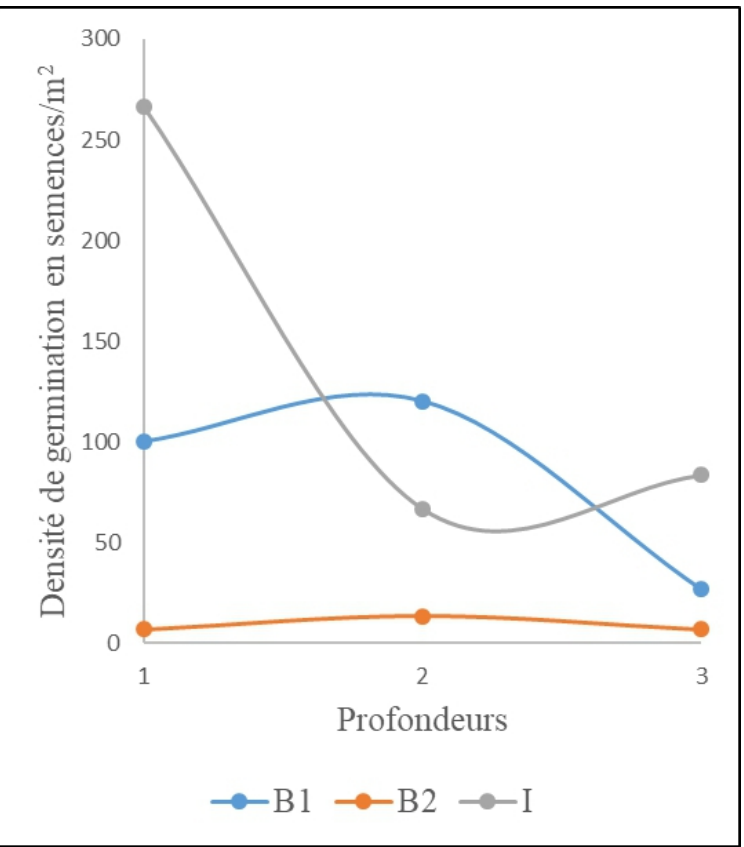

Figure 4 : Variation des densités de germination par provenance et par strate de sol

D'après le tableau 1 et la figure 4 , le prélèvement du sol forestier dans la première couche du sol de la plantation (sous couvert végétal) donne la densité de germination la plus élevée. Si l'on doit faire un prélèvement dans la bande 1, ou dans la bande 2, la meilleure couche est celle de la profondeur $2(5-10 \mathrm{~cm})$. Plus, on s'éloigne de la plantation, moins efficace est le sol pour conserver les semences de Acacia auriculiformis, et la couche intermédiaire $(5-10 \mathrm{~cm})$ devient le lieu idéal de conservation de ces semences dans le sol. 
Les résultats de la régression logistique ont montré que seule la provenance influence significativement la viabilité des semences issues de la banque de graines du sol. Il en ressortait que, le prélèvement dans la plantation augmente significativement par rapport aux autres provenances, la viabilité des semences $(\operatorname{Pr}(>|z|)=0,00718)$. De plus, la provenance B1 améliore mieux que la provenance B2 cette viabilité, même si cette amélioration n'est pas statistiquement significative $(\operatorname{Pr}(>|z|)=0,26042)$. L'interaction entre les deux facteurs ne s'est pas révélée améliorer la viabilité des semences. Le tableau 2 ci-dessous présente les résultats du modèle.

Tab2. Influence de la viabilité de germination par la provenance

\begin{tabular}{crrrrr}
\hline & \multicolumn{1}{c}{ Estimate } & \multicolumn{1}{c}{ Std, Error } & \multicolumn{1}{c}{$\mathrm{z}$ value } & \multicolumn{1}{c}{$\operatorname{Pr}(>|\mathrm{z}|)$} & Signification \\
\hline (Intercept) & $-0,1335$ & 0,5175 & $-0,258$ & 0,7964 & \\
\hline ProvenanceB2 & $-0,8781$ & 0,7802 & $-1,125$ & 0,26042 & \\
\hline ProvenanceI & 2,0053 & 0,7459 & 2,689 & 0,00718 & $* *$ \\
\hline
\end{tabular}

La régression négative binomiale de Poisson s'est révélée adéquate aux données avec une probabilité d'adéquation de 0,1467 . Elle a montré que la profondeur et la provenance influencent significativement la performance de germination des semences issues de la banque de graines du sol. D'une part, les prélèvements dans la plantation ou dans la première bande $(10 \mathrm{~m}$ de la lisière) offrent les meilleures performances de germination en augmentant le nombre moyen de semences qui donnent des plantules de Acacia auriculiformis. D'autre part, il est remarqué que la première couche de sol (0$5 \mathrm{~cm}$ ) de la plantation représente la meilleure option. Le tableau 3 ci-dessous présente les résultats du modèle.

Tab3. Influence de la performance de germination par la provenance et la profondeur

\begin{tabular}{lrrrrc}
\hline & \multicolumn{1}{c}{ Estimate } & \multicolumn{1}{c}{ Std, Error } & \multicolumn{1}{c}{$\mathrm{z}$ value } & \multicolumn{1}{c}{$\operatorname{Pr}(>|\mathrm{z}|)$} & Signification \\
\hline (Intercept) & 1,432 & 0,358 & 4 & $6,33 \mathrm{E}-05$ & $* * *$ \\
\hline ProvenanceB2 & $-2,215$ & 0,6312 & $-3,509$ & 0,000449 & $* * *$ \\
\hline ProvenanceI & 0,4433 & 0,359 & 1,235 & 0,216907 & \\
\hline ProfondeurP2 & $-0,684$ & 0,3761 & $-1,819$ & 0,068949 & . \\
\hline ProfondeurP3 & $-1,0994$ & 0,3928 & $-2,799$ & 0,005128 & $* *$ \\
\hline
\end{tabular}

L'interaction entre les deux facteurs dans ce cas s'est révélée avoir un effet significatif $(\operatorname{Pr}(>|z|)=0,0472)$ sur la performance de germination. Il ressortait de l'analyse que les prélèvements dans la première couche de la plantation offraient la meilleure performance de germination. Le tableau 4 cidessous présente les résultats du modèle.

Tab4. Influence de l'interaction entre provenance et profondeur sur la performance de germination

\begin{tabular}{lrrrrc}
\hline & Estimate & Std,Error & \multicolumn{1}{c}{ zvalue } & $\operatorname{Pr}(>|z|)$ & Signification \\
\hline (Intercept) & 1,0986 & 0,4618 & 2,379 & 0,0174 & $*$ \\
\hline ProvenanceB2 & $-2,7081$ & 1,1662 & $-2,322$ & 0,0202 & $*$ \\
\hline ProvenanceI & 0,9808 & 0,5469 & 1,793 & 0,0729 & $*$ \\
\hline ProfondeurP2 & 0,1823 & 0,6446 & 0,283 & 0,7773 & \\
\hline
\end{tabular}




\begin{tabular}{lrrrrr}
\hline ProfondeurP3 & $-1,3218$ & 0,781 & $-1,692$ & 0,0906 & $*$ \\
\hline ProvenanceB2:ProfondeurP2 & 0,5108 & 1,4862 & 0,344 & 0,7311 & $*$ \\
\hline ProvenanceI:ProfondeurP2 & $-1,5686$ & 0,7903 & $-1,985$ & 0,0472 & $*$ \\
\hline ProvenanceB2:ProfondeurP3 & 1,3218 & 1,7039 & 0,776 & 0,4379 & \\
\hline ProvenanceI:ProfondeurP3 & 0,1586 & 0,8995 & 0,176 & 0,86 &
\end{tabular}

La densité de germination obtenue pour la meilleure combinaison ( 0 $5 \mathrm{~cm}$ dans la plantation) est de 267 semences par $\mathrm{m}^{2}$ et appartient à l'intervalle définit par Garwood (1989) pour les sols tropicaux, bien que, dans notre cas d'étude, c'est seulement les plantules de Acacia auriculiformis obtenues qui soient comptées. Cette valeur certes, ne fait pas partie des moindres pour la production de plants mais est inférieure à beaucoup d'autres obtenues par des études antérieures (Hall \& Swaine, 1980 ; Daïnou et al., 2011 ; Sousa et al., 2017) portant sur des peuplements naturels plurispécifiques, ce qui est tout à fait normal puisque quelques autres espèces auraient pu augmenter cette densité si leurs plantules étaient comptées. Néanmoins, le niveau élevé de densité obtenue pourrait être expliqué par la structure du peuplement (peuplement équienne mono spécifique de Acacia auriculiformis) puisque les résultats de Dalling \& Denslow (1998) révélaient que la densité de la banque de semences du sol est corrélée à l'abondance des espèces formant cette banque de semences. Cependant, il est possible que le mode de gestion du peuplement en place fasse que seulement une faible proportion de cette banque de semences provienne des espèces présentes (Decocq et al., 2004). Notre étude ne s'est pas intéressée aux autres semences d'autres espèces qui pourraient être présentes dans le sol. Les résultats pourraient aussi varier en fonction des milieux et en fonction de l'âge des plantations.

Dans le sous-bois de la plantation de Acacia auriculiformis, la première couche allant de 0 à $5 \mathrm{~cm}$ de profondeur conserve mieux les semences. Ceci pourrait s'expliquer par la grande quantité de production de biomasse par l'espèce (Fonton et al., 2002b). La biomasse, faisant partie du sol, devrait contribuer à la protection des semences. Il est aussi important d'ajouter que les semences qui se retrouvent dans cette strate du profil pédologique seraient les dernières à tomber et de ce fait devraient être fortement viables, sauf exceptions liées aux paramètres biologiques intrinsèques de l'espèce. Dans les bandes 1 et 2, c'est la profondeur 2 qui protège mieux les semences de l'espèce parce que l'ouverture du couvert dans ces bandes ne facilite pas une bonne conservation des semences dans la première strate du profil pédologique, et c'est aussi à comprendre que le temps de séjour des semences depuis le jour de la chute pour appartenir à la deuxième strate du profil pédologique est relativement plus court que celui des semences qui se trouvent dans la troisième strate du profil pédologique. Il est aussi possible que les semences qui atteignent le sol soient enclines à perdre leur 
viabilité avant la constitution d'une épaisseur de sol de 10 à $20 \mathrm{~cm}$. Le facteur «provenance » met en jeu le couvert végétal et la quantité de litière dans la conservation alors que le facteur «profondeur » met beaucoup plus en jeu le facteur temps. La banque de semence du sol est influencée par la situation par rapport au peuplement et par le couvert végétal (Augusto et al., 2001). Nos résultats confirment ceux de ces auteurs. Les bandes 1 et 2 étant hors de la plantation, pourraient offrir moins de protections aux semences qui y tombent d'où les faibles densités de germination obtenues dans lesdites bandes. Quelques semences sont disséminées hors des peuplements, et lorsque cela se passe, leur viabilité est précaire. Cette faible protection par le sol serait accentuée par l'aménagement de la forêt à but touristique. De plus, la présence des habitations et infrastructures à la périphérie immédiate de la plantation où le vent pourrait éventuellement disséminer les graines semble aussi cautionner cette inefficacité des bandes 1 et 2 . Le taux de prédation serait probablement élevé (Cubiña \& Aide, 2006) à cause d'une absence de protection, ce qui explique la variation observée entre provenances. Ainsi, plus on s'éloigne de la plantation, moins efficace est la banque de semences du sol.

\section{Conclusion}

Cette étude aboutit à la conclusion que le sol sous couvert végétal de Acacia auriculiformis conserve dans sa couche inférieure, les semences de l'espèce. En conséquence, il serait plausible de produire à partir du sol des plantations de Acacia auriculiformis, des plantules de l'espèce sans avoir à appliquer des prétraitements, ni chercher péniblement et en vain, les semences de l'espèce. Les présents résultats permettront de remédier aux problèmes liés à la fourniture de plants pour les reboisements. Cependant, la présente étude n'est pas un prétexte pour éroder les sols des plantations de Acacia auriculiformis. Tout prélèvement doit faire objet de consultation des services forestiers scientifiques compétents. Nous envisageons de répéter l'expérimentation dans diverses plantations afin d'avoir des éléments de comparaison dans nos conditions écologiques.

\section{References:}

1. Adomou, A.C. (2005). Vegetation Patterns and Environmental Gradients in Benin: Implications for Biogeography and Conservation, Wageningen Universiteit.

2. Akouehou, G., Duclos, L., Able, C. (2009). Rapport sur Instruction d'Inventaire et Actualisation des cartes parcellaires des Plantations Domaniales de Bois de Feu. PBF-II. BAD. 62 p.

3. Akpona, T.J.D., Idohou, R., Assogbadjo, A., Salako, K.V., Glèlè Kakaï, R. (2016). History, Impact of Soil Types on Stand Structure and Growth of the Dry Zone Mahogany (Khaya senegalensis (Desr,) 
A,Juss,) in Plantation in Benin (West Africa), Environment and Ecology Research 4(4): 193-199.

4. Augusto, L., Dupouey, J-L., Picard, J-F., Ranger, J. (2001). Potential contribution of the seed bank in coniferous plantations to the restoration of native deciduous forest vegetation. Acta Oecologica 22, $87-98$.

5. Aveyard, J.M. (1968). The effect of seven pre-sowing treatments on total germination and germination rate of six Acacia species, Journal of the Soil Conservation Service of New South Wales, 24, 43-54,

6. Bauduin, A. (2009). Etude du potentiel séminal édaphique de peuplements à Pericopsis elata (Harms) van Meeuwen (assamela) au sud-est du Cameroun, Travail de fin d'études En vue de l'obtention du grade de Bioingénieur en : Nature, Eaux et Forêts, Faculte Universitaire Des Sciences Agronomiques de Gembloux, Belgique ; $139 \mathrm{p}$.

7. Brown, N.A.C., Booysen, P. de V. (1969). Seed coat impermeability in several Acacia species, Agroplantae 1, 51-60,

8. Clemens, J., Jones, P.G., Gilbert, N.H. (1977). Effect of Seed Treatments on Germination in Acacia, Australian Journal of Botany, 25, 269-76.

9. Cubiña, A., Aide, T.M. (2006). The Effect of Distance from Forest Edge on Seed Rain and Soil Seed Bank in a Tropical Pasture. BlOTROPlCA 33(2): 260-267.

10. Daïnou, K., Bauduin, A., Bourland, N., Gillet, J.F., Fétéké, F., Doucet, J-L. (2011). Soil seed bank characteristics in Cameroonian rainforests and implications for post-logging forest recovery. Ecol. Eng. 37, 1499-1506.

11. Dalling, J.W., Denslow, J.S. (1998). Soil seed bank composition along a forest chronosequence in seasonally moist tropical forest, Panama. Journal of Vegetation Science 9: 669-678.

12. Decocq, G., Valentin, B., Toussaint, B., Hendoux, F., Saguez, R., Bardat, J. (2004). Soil seed bank composition and diversity in a managed temperate deciduous forest. Biodiversity and Conservation 13: 2485-2509

13. Douh, C., Daïnou, K., Loumeto, J.J., Moutsambote, J-M., Fayolle, A., Tosso, F., Forni, E., Gourlet-Fleury, S., Doucet, J-L. (2018). Soil seed bank characteristics in two central African forest types and implications for forest restoration. Forest Ecology and Management 409, 766-776.

14. Fonton, N.H., Glele Kakai, R., Rondeux, J. (2002a). Étude dendrométrique d'Acacia auriculiformis A, Cunn, ex Benth, en 
mélange sur vertisol au Bénin, Biotechnology, Agronomy, Society and Environment, 20026 (1), 29-37.

15. Fonton, N.H., Claustriaux, J.J., Agbahungba, G. (2002b). Performance de Acacia auriculiformis (Cunn, A,) dans le système agroforestier au Sud-Bénin, Annales des Sciences Agronomiques du Bénin (3)1 :65-79,

16. Ganglo, J.C., de Foucault, B. (2006). Plant communities, forest site identification and classification in Toffo reserve, South-Benin, Bois et Forêts des Tropiques, 288 (2): 25-38.

17. Garwood, N.C. (1989). Tropical soil seed banks: a review. In: Leck, M.A., Parker, V.T., Simpson, R.L. (Eds.), Ecology of Soil Seed Banks. Academic Press, San Diego, CA, pp. 149-209.

18. Gnahoua, G.M., Louppe, D. (2003). Acacia auriculiformis. Fiche technique. <cirad00429281>

19. Hall, J.B., Swaine, M.D. (1980). Seed stocks in Ghanaian forest soils. Biotropica 12, 256-263.

20. HarvestChoice, (2010), Agro-ecological Zones of Sub-Saharan Africa, International Food Policy Research Institute, University of Minnesota, Washington, DC.

21. ICRAF, (2006). Bonnes pratiques de culture en pépinière forestière Directives pratiques pour les pépinières de recherche, Manuel technique no, 3, World Agroforestry Center, Nairobi, Kenya, PDF, 93 p.

22. IPCC, (2007). IPCC Fourth Assessment Report, Working Group I Report "The Physical Science Basis", Section 7,3,3,1,5 (p, 527).

23. Myers, A. (1936). The germination of Cootamundra wattle and other Acacia seed, Agricultural Gazette of New South Wales, 47, 55.

24. Neuenschwander, P., Sinsin, B., Goergen, G. (2011). Protection de la Nature en Afrique de l'Ouest: Une Liste Rouge pour le Bénin Nature Conservation in WestAfrica: Red List for Benin, Ibadan, IITA.

25. Opuni-Frimpong, E., Karnosky, D.F., Storer, E.A., Cobbinah, J.R. (2008). Silvicultural systems for plantation mahogany in Africa: influences of canopy shade on tree growth and pest damage, Forest Ecology and Management 255, 328-333.

26. PAP, (2010). Plan d'Aménagement et de Gestion Participatif de la forêt classée de Pahou, 121p.

27. Perera, G.A.D. (2005). Spatial heterogeneity of the soil seed bank in the tropical semi deciduous forest at Wasgomuwa National Park, Sri Lanka, Tropical Ecology 46 (1), 79-89.

28. R Core Team, (2016). R: A language and environment for statistical computing. RFoundation for Statistical Computing. 
29. Schweinbacher, E., Marcante, S., Erschbamer, B. (2010). Alpine species seed longevity in the soil in relation to seed size and shape-A 5-year burial experiment in the Central Alps. Flora (Jena) 205, 19-25.

30. Sinsin, B., Matig, O.E., Assogbadjo, A., Gaoué, O., Sinadouwirou, T. (2004). Dendrometric characteristics as indicators of pressure of Afzelia africana Sm, dynamic changes in trees found in different climatic zones of Benin, Biodiversity \& Conservation, 138 : 15551570.

31. Sousa, T.R., Costa, F.R.C., Bentos, T.V., Leal Filho, N., Mesquita, R.C.G., Ribeiro, I.O. (2017). The effect of forest fragmentation on the soil seed bank of Central Amazonia. Forest Ecology and Management. 393, 105-112.

32. Swaine, M. (2001). Protocol for assay of soil seed banks, http://www,nbu,ac,uk/tropical/SSBprotocol_Swaine,doc,, consulté le 24 juin 2008.

33. Volkoff, B., Willaime, P. (1976). Carte pédologique de reconnaissance de la République Populaire du Bénin à 1/200 000: feuille de PortoNovo.

34. Waylen, K. (2006). Botanic Gardens: using biodiversity to improve human well-being, Botanic Gardens Conservation International, Richmond, UK, 32p.

35. White, F. (1986). La Végétation de L'Afrique (The Vegetation of Africa), IRD Editions.

36. Zakra, N., Domenach, A.M., Sangara, A. (1996). Positive result of coconut/acacia intercropping for nitrogen, potassium and magnesium restoration, Plantation-Recherche-Developpement, 3(1):39-48. 